\title{
La perizia criminologica attraverso l'analisi di un caso
}

\author{
Augusto Balloni
}

\begin{abstract}
Riassunto
L'autore propone alcune riflessioni a partire da un'esperienza di consulenza tecnica d'ufficio che gli è stata affidata sul caso Cogne. Si esaminerà la vicenda processuale nella fase esecutiva in chiave ermeneutica criminologica, vale a dire attraverso il tentativo di commentare e di reinterpretare un'attività di consulenza nella prospettiva della perizia criminologica, mai formalmente ammessa, ma con possibilità di utilizzarne gli schemi operativi come si è verificato nel caso che qui viene riproposto. Infatti, si vuole, con queste riflessioni, approfondire il dibattito relativo a criminologia, vittimologia e sicurezza. In effetti, l'autore, nel ripercorrere la traccia della consulenza tecnica d'ufficio e le ordinanze del Tribunale di Sorveglianza di Bologna, analizza il delitto, la sua autrice e la possibilità di valutarne il futuro comportamento, senza trascurare la vittima e le questioni di difesa sociale, intese come aspetti della sicurezza volti ad evitare il rischio del ripetersi di condotte devianti o antigiuridiche.
\end{abstract}

\section{Résumé}

L'auteur propose quelques pistes de réflexions à partir de l'expertise médico-légale et psychiatrique sur «l'affaire Cogne » par lui rédigée et qui lui a été demandée par le Tribunal de l'application des peines (Tribunale di Sorveglianza) de Bologne.

Il examinera les documents de la phase procédurale de l'application de la peine selon le point de vue herméneutique criminologique, c'est-à-dire en essayant de commenter et de réinterpréter une activité de consultation dans la perspective de l'expertise criminologique. Cette dernière n'est jamais admise formellement, mais il existe la possibilité de se servir de ses schémas opérationnels, comme dans le cas ici proposé.

En effet, par ces réflexions, l'auteur veut élargir le débat concernant la criminologie, la victimologie et la sécurité. En fait, en suivant les traces de l'expertise et des arrêtés du Tribunal de l'application des peines de Bologne, l'auteur analyse le crime, la coupable et il n'exclut pas la possibilité d'évaluer son comportement futur. Bien évidemment, il ne néglige pas la victime et les questions de défense sociale, interprétées comme autant d'aspects de la sécurité ayant pour but d'éviter le risque de répétition de comportements déviants ou anti-juridiques.

\section{Abstract}

The author offers some thoughts based on the forensic and psychiatric expert's report on the so-called "Cogne case" written by himself and requested by the Surveillance Court of Bologna (Tribunale di Sorveglianza).

He will examine the documents of the proceedings regarding the sentencing phase from a hermeneutic criminological perspective, as to say by intending to comment and reinterpret a consultation activity in view of criminological expertise. This last report has never been formally admitted, but it is possible to use its operational schemata, as is the case here.

In fact, the author, with these thoughts, aims to expand the debate concerning criminology, victimology and security. Undoubtedly, by following the paths of the export's report and the decisions of the Surveillance Court of Bologna, the author analyses the crime, the individual who was found guilty and he does not rule out either the possibility of evaluating the future behaviour of this person. Obviously, he does not intend to overlook the victim or any social defence issues, interpreted as aspects of security aiming to avoid the risk of re-occurence of deviant or anti-juridical behaviours.

Key words: Cogne case; forensic and psychiatric expert's report; criminology; victimology; security.

\section{Introduzione.}

Il "caso Cogne" ha suscitato interesse perché si che è stata condannata a 16 anni di reclusione per riferisce alla vicenda processuale di una mamma l'omicidio di un figlio. Parallelamente al processo giudiziario, vi è stato un vero e proprio processo

\footnotetext{
- Neuropsichiatra, medico legale, psicologo, già professore ordinario di Criminologia all'Università di Bologna, è stato nominato consulente tecnico d'ufficio dal Tribunale di Sorveglianza di Bologna in data 10.12.2013 e in questo articolo si propongono alcune riflessioni sul cosiddetto "Caso Cogne".
} 
mediatico che ha coinvolto molti esperti o sedicenti esperti di criminologia o di materie affini. È però un dato di fatto che questa vicenda giudiziaria ha veramente coinvolto esperti medico-legali, psichiatri, psicologi e criminologi.

Il contributo di questi esperti ha stimolato una rivisitazione di alcuni aspetti di questa vicenda.

Prima però di ripercorrere il caso in esame, verranno svolte alcune considerazioni in tema di progettazione: infatti, volendo far riferimento all'ultima consulenza che ha riguardato AMF, occorre precisare che ogni perizia o consulenza può rivestire il significato di un progetto che può inserirsi in uno di quei più ampi progetti che riguardano i temi operativi dell'amministrazione della giustizia.

Il "caso Cogne" è giunto ormai alla fase esecutiva, tuttavia continua a suscitare notevole attenzione senza però che spesso traspaia il vero significato degli accertamenti disposti dal Tribunale di Sorveglianza di Bologna. In effetti, questo tribunale ha dato avvio ad una consulenza criminologica-psichiatrica, orientando gli accertamenti verso le discipline criminologiche, psichiatriche e socio-psicologiche e facendo sì che si realizzasse l'impiego della perizia criminologica, così come è consentito nella sola fase dell'esecuzione e come è previsto dall'art. 220 comma 2 c.p.p.

In relazione a ciò, il Tribunale di Sorveglianza di Bologna, dopo aver valutato preliminarmente i profili oggettivi dell'ammissibilità dell'istanza di detenzione domiciliare speciale ex art. 47 quinquies L. n. 354/75 avanzata da AMF, esamina le condizioni di merito che qui di seguito si riportano integralmente: “[...] Rileva il tribunale come la norma in esame richieda, ai fini dell'ammissione alla misura, che non sussista 'un concreto pericolo di commissione di ulteriori delitti', e connota detta prognosi di non recidività ad ulteriori delitti dei quali si vuole evitare la commissione, da intendersi per quelli della medesima specie, di quelli per i quali è in esecuzione la pena.

Orbene, le consulenze tecniche e psichiatriche, disposte nei due gradi di giudizio, hanno escluso la pericolosità dell'allora imputata, intesa come rischio di commissione di ulteriori condotte delittuose. Tuttavia, la consulenza tecnica collegiale, disposta durante il primo grado di giudizio, peraltro la sola alla quale l'imputata abbia collaborato, rivela come 'si tratta di soggetto che presenta un funzionamento psichico sufficientemente adeguato, capace di controllo di realtà $\quad . . \quad$ Su questa condizione di equipaggiamento psichico di base, si osserva una situazione critica di forte distanziamento difensivo dal coinvolgimento emotivo verso situazioni nuove, che comporta una rigidità a livello cognitivo, ed una regressione alla dipendenza nella gestione delle emozioni e degli affetti, a protezione di un sé vitale ...' (v. pag. 45).

La consulenza tecnica d'ufficio, disposta nel corso del giudizio di appello, ritiene inoltre che, al momento del fatto, l'imputata fosse affetta da sindrome ansiosa in soggetto con assetto di personalità connotato da prevalenti componenti di tipo isterico.

Occorre osservare come gli elaborati peritali presi in esame, se pur contenenti importanti analisi sulla personalità dell'istante, siano tuttavia stati redatti in un'epoca oramai remota, essendo trascorsi già sette anni dalla stesura di quella del secondo grado e oltre dieci da quella del primo grado di giudizio. 
Si ritiene, pertanto, non possano essere assunti come elementi probanti ai fini della valutazione del parametro normativo relativo all'assenza di pericolo di recidiva.

A tal fine, dovrebbe soccorrere, anche se i fini di un giudizio più ampio sulla pericolosità della condannata, la relazione di sintesi di osservazione scientifica della personalità, redatta da Gruppo di osservazione e trattamento dell'Istituto penitenziario. Detto elaborato è previsto, come è noto, dall'art. $13,2^{\circ}$ co., O.P., che dispone 'Nei confronti dei condannati e degli internati, è disposta l'osservazione scientifica della personalità per rilevare le carenze fisio-psichiche e le altre cause del disadattamento sociale. L'osservazione è compiuta dall'inizio dell'esecuzione e proseguita nel corso di essa'. Le modalità dell'osservazione scientifica della personalità vengono poi esplicitate dall'art. 27 del D.P.R. n. 230/2000, contenente il regolamento di esecuzione della legge penitenziaria. Si osserva, a tale proposito, come la norma, contenuta nel capo III, recante le 'Modalità del trattamento', trovi applicazione in relazione ad ogni misura alternativa, compresa quella richiesta, della detenzione domiciliare speciale.

La relazione di osservazione della Casa Circondariale di Bologna, in atti, non appare assolutamente illuminante sul piano dell'analisi della personalità della condannata, tanto che vi si afferma esplicitamente come sia stato privilegiato l'aspetto trattamentale rispetto a quello dell'osservazione personologica.

Detta reazione evidenzia quasi esclusivamente le notevoli difficoltà incontrate dalla detenuta, sin dall'inizio della carcerazione, con assunzione, nelle prime fasi, di terapia farmacologica ed assidui colloqui con l'esperto ex art. 80 O.P. Evidenzia poi che la donna ha manifestato sentimento di angoscia e disperazione per aver subito una doppia ingiustizia: la morte violenta del figlio e la condanna per un atto che non ha compiuto.

Il lavoro dell'esperto viene delimitato, come è esplicitato nella relazione, al "cercare di adattare la donna, che tuttora presenta sentimento di tristezza, di rabbia e di timore per il futuro, alla realtà detentiva e alla nuova realtà personale e familiare'.

La relazione ripercorre, inoltre, le condizioni familiari della detenuta e le attività svolte all'interno dell'Istituto. Sulla personalità della condannata, nulla o quasi viene detto che possa supportare il giudizio prognostico, in questa sede richiesto, sul pericolo di recidiva o, quantomeno, sui profili attuali della personalità e sulla progressione trattamentale in relazione, anche approssimativa, con condotte future, fermandosi gli esperti penitenziari alla proclamazione di innocenza.

Questo collegio, ritenuto pertanto insufficiente, per un verso, l'espletata osservazione della personalità, e per altro verso, inutile un proseguo di osservazione in dette forme, alla luce degli elementi esposti, reputa necessario, al fine della decisione, disporre un accertamento tecnico di ufficio sulla personalità dell'istante, ai sensi dell'art. 220, $2^{\circ}$ co., c.p.p., a mente del quale: 'Salvo quanto previsto ai fini dell'esecuzione della pena e della misura di sicurezza, non sono ammesse perizie per stabilire l'abitualità o la professionalità del reato, la tendenza a delinquere, il carattere e la personalità dell'imputato e in 
genere le qualità psichiche indipendenti da cause patologiche'.

Appare chiaro dal dettato normativo come, in sede di esecuzione di procedimento di sorveglianza, possono essere ammesse perizie sul carattere e sulla personalità del condannato" .

Per questi motivi, il Tribunale di Sorveglianza ha disposto consulenza tecnica di ufficio nei riguardi della condannata nominando un consulente tecnico di ufficio e fissando un'udienza per il conferimento dell'incarico e per la formulazione dei quesiti.

Prima di procedere nell'analisi del caso, appare opportuno esporre alcune considerazioni in tema di perizia e di consulenza ${ }^{2}$.

La perizia psichiatrica, ad esempio, si inserisce nel processo penale come atto istruttorio o anche come prova. Essa però, tra tutte le perizie, ha una posizione particolare, perché non serve mai a provare che un fatto sussiste o non sussiste, ma serve piuttosto ad accertare quale sia lo stato di mente di un individuo nel momento in cui commise il fatto per cui è imputabile. In particolare, ai sensi dell'art. 85 c.p., perché un soggetto sia imputabile, processabile e punibile, occorre che sia in possesso delle capacità di intendere e/o di volere. Oltre alla perizia

1 Dall'ordinanza del Tribunale di Sorveglianza depositato in cancelleria il 12.12.2013, composto da: Dott. Francesco Maisto - Presidente, Dott. Sabrina Bosi - Magistrato di Sorveglianza est. - Dott. Raffaella Sette - esperta, Dott. Cinzia Bertuccioli - esperta.

${ }^{2}$ Balloni A., Criminologia e psicopatologia. Analisi di 110 perizie psichiatriche, Patron, Bologna, 2004; Balloni A., "La perizia psichiatrica: problemi, ipotesi e prospettive", in Balloni A., (a cura di), Vittime Crimine Difesa Sociale, Clueb, Bologna, 1989, pp. 51-73; Balloni A., "Nuove metodologie nell'indagine peritale con riferimento alla psicopatologia", in Lombardi M.A. (a cura di), La Perizia e la Consulenza tecnica: stato attuale e prospettive, Pacini Editore, Pisa, 1990; Balloni A., "Psichiatria forense", in Pancheri P. e psichiatrica, vi sono molti altri tipi di perizie, che si inseriscono nella dinamica processuale, come ad esempio, la perizia necroscopica, che può servire a provare se una morte sia dovuta ad omicidio, o a suicidio, od a un fatto accidentale non interessante l'amministrazione della giustizia. Inoltre si richiedono perizie in materia di responsabilità professionale, in ambito urbanistico, nel settore degli infortuni sul lavoro e in molti altri settori.

In effetti, la perizia è ammessa ogni qualvolta occorra svolgere indagine $\mathrm{o}$ acquisire dati $\mathrm{o}$ valutazioni che richiedono specifiche competenze tecniche, scientifiche (art. 220 c.p.p.) o artistiche. Allo stesso modo, "Quando è necessario, il giudice può farsi assistere, per il compimento di singoli atti o per tutto il processo, da uno o più consulenti di particolare competenza tecnica (...)" (art. 61 c.p.c.). La diversa terminologia (consulenza e perizia) non controindica a trattare in modo unitario le questioni collegate alla funzione e alla struttura della perizia o consulenza in qualsiasi sede (civile, penale, tribunale di sorveglianza) si manifesti la necessità di avvalersi dell'opera (consulenza) di una persona fornita di particolare competenza (perito) in particolari discipline e, nel caso specifico, nell'ambito psicologico e psichiatrico.

È evidente che l'aspetto unificante della perizia e della consulenza è la competenza che - insieme ad altre caratteristiche quali: l'ufficialità (entrambe vengono disposte d'ufficio), rispetto del principio del contraddittorio (le parti possono farsi assistere dai propri consulenti), e la libera valutazione dei risultati da parte del giudice (peritus peritorum) concorre a dotare la consulenza e la perizia di

Cassano G.B. (coordinato da), Trattato italiano di 
caratteristiche comuni. In particolare, il perito e il consulente d'ufficio devono essere, anche in psichiatria forense, psicologia giudiziaria $\mathrm{o}$ criminologia psichiatrica, dotati di particolari o specifiche competenze tecniche per svolgere la loro opera che molto spesso si caratterizza come una progett-azione che si realizza in termini operativi per realizzare appunto quel progetto, la relazione di perizia o di consulenza, con cui si comunica il prodotto di una particolare conoscenza da parte di colui che la possiede a colui che (giudice o avvocato), non possedendola, $\mathrm{o}$ non possedendola in modo completo $\mathrm{o}$ approfondito, o anche imperfetto, deve utilizzare questo prodotto in una o più decisioni operative.

Infatti la perizia o la consulenza costituiscono atti concreti di una progettazione che è una serie logica e precisa di operazioni con le quali si da ordine, sistematicità e organicità al lavoro svolto che, nel caso in esame ("caso Cogne") dovrà essere inserito nel processo-progetto rieducativo. In una tal prospettiva, il perito incontra nella realizzazione del suo progetto diversi attori nei rispettivi ruoli: il giudice o il tribunale che gli dà l'incarico, gli appartenenti alla struttura penitenziaria (agenti penitenziari, medici del carcere, educatori, psicologi, staff dirigenziale della struttura penitenziaria, assistenti sociali, ....); d'altra parte, il perito o il consulente realizzano il proprio progetto in un particolare contesto: l'ambiente carcerario o il proprio studio professionale. Nell'ambito del progetto con sui si configura e poi si realizza la perizia o la consulenza, occorre dare un corretto spazio alle parti perché possano partecipare adeguatamente alle operazioni peritali. Perciò, il consulente o il

psichiatria, Masson, Milano, 1999. perito per realizzare il proprio progetto (relazione di perizia o di consulenza) deve essere in grado di saper elaborare una valutazione tecnico-legale dei fatti e delle procedure, con l'auspicio che l'operato dei consulenti delle parti possa essere improntato a collaborazione senza che vi siano ostacoli alla realizzazione del progetto.

Nella realizzazione di questo progetto, il colloquio $^{3}$ è un elemento importante perché con esso si tende a realizzare un'adeguata conoscenza dell'altro per la formulazione dei giudizi sulla personalità, sui fatti accaduti o su altre caratteristiche che possono incidere sul comportamento.

Si realizza in tal modo un approccio dialogico che M. Bachtin, il pensatore russo nei suoi studi sulla metodologia delle scienze umane, propone come interazione dialogica, mettendo "in luce una serie di problemi cruciali per la comprensione dell'Altro. Innanzitutto egli sottolinea l'inesauribilità della conoscenza personale a cui si lega però la possibilità di un approfondimento progressivo. 'Il criterio qui non è l'esattezza della conoscenza, ma la profondità della penetrazione [...] l'importanza di pervenire ed addentrarsi fino al nucleo creativo della persona [...] il penetrare nell'altro (il fondersi con esso) e il mantenere la distanza (il proprio posto) che garantisce l'eccedenza di conoscenza. Nelle scienze umane, l'esattezza è il superamento dell'alterità altrui senza trasformarlo nel proprio [...] la comprensione come trasformazione dell'altrui nel proprio-altrui'.

\footnotetext{
${ }^{3}$ Balloni A., "Colloquio, test mentali e psicopatologia", in Guidicini P. (a cura di), Nuovo manuale della ricerca sociologica, Franco Angeli, Milano, 1981, cap. 14, pp. 339-351; Balloni A., Nathan F., "Il colloquio clinico nella perizia psichiatrica", Giornale $D i$ Medicina Legale Infortunistica E Tossicologia, Suppl.
} 
Inoltre è necessario realizzare un'atmosfera di benevolenza e simpatia. ...

'Il discorso intimo è compenetrato da una profonda fiducia nel destinatario, nella sua simpatia, nella sensibilità e benevolenza della comprensione responsiva. In questa atmosfera di profonda fiducia, il parlante svela le proprie profondità interiori (1988b, p. 287)'.

Il concetto del proprio-altrui significa che la conoscenza dell'altro è inevitabilmente influenzata dai parametri concettuali e linguistici del conoscente. Nella misura in cui abbiamo coscienza di questi parametri, esercitiamo il massimo di rispetto possibile per l'alterità dell'altro. La posizione bachtiana è in linea con gli aspetti delle varie scuole contemporanee: della fenomenologia, della scuola ermeneutica e della psicoanalisi. La fenomenologia richiama al rispetto per la personalità dell'altro e alla necessità di collocarsi nel suo orizzonte concettuale. ...

L'ermeneutica sottolinea l'impossibilità di uscire dal circolo ermeneutico e di abbandonare le proprie precomprensioni; è necessario tuttavia prendere coscienza di queste precomprensioni considerandole anche come utili congetture, ipotesi da vagliare. Sulla stessa linea, si collocano le scuole psicoanalitiche, quando prescrivono che il terapeuta, mediante l'analisi personale, prenda coscienza dei propri problemi prima di affrontare quelli del paziente"

In una tal prospettiva, anche nell' ambito peritale, dove possono realizzarsi attività che hanno funzioni terapeutiche, è necessario che il perito,

al n. 4,1968, pp. 636-644.

${ }^{4}$ Galli G. (a cura di), La persona in relazione. Sviluppi della psicologia della Gestalt, Liguori Editore, Napoli, 2009, pp. 34-35. soprattutto attraverso l'esperienza, abbia coscienza e consapevolezza dei propri problemi per non compromettere il valore e il significato dei contenuti del colloquio. Infatti è evidente che con il colloquio si cerca di realizzare una conoscenza dell'altro per la formulazione di giudizi e anche per mettere in evidenza caratteristiche latenti.

Fatte queste premesse, si precisa che il "caso Cogne" si apre con quelle indagini medico-legali che riguardano l'omicidio in cui si deve individuare attraverso l'esame del cadavere e la successiva autopsia, le cause della morte, l'epoca della morte e tutte quelle circostanze rilevanti ai fini dell'indagine.

In seguito, si sono svolti numerosi accertamenti riguardanti la capacità di intendere e di volere dell'imputata e la sua pericolosità sociale attraverso diverse perizie psichiatriche. In una tale ottica, sono diventati significativi i rapporti tra malattie mentali e condotte criminose che si collegano strettamente alla perizia psichiatrica e quindi impongono di segnalare le linee guida secondo cui deve svolgersi tale perizia e che sono fissate negli articoli dal 220 al 233 del codice di procedura penale. In particolare, nel comma 2 dell'art. 220 c.p.p., già segnalato, si sottolinea, come del resto era previsto dall'art. 314 c.p.p. abrogato, la non ammissibilità della perizia per stabilire l'abitualità o la professionalità nel reato, la tendenza a delinquere, il carattere e la personalità dell'imputato e in genere le qualità psichiche indipendenti dalle cause patologiche, salvo quanto previsto ai fini della pena o delle misure di sicurezza. È quindi evidente che nella fase dell'esecuzione della pena si può appunto fare riferimento alla possibilità di disporre una 
perizia su un soggetto in espiazione di pena per la valutazione della sua personalità. Infatti, ai fini della pena, intervengono i provvedimenti del tribunale di sorveglianza per cui, allorché si procede nei confronti di persona sottoposta ad osservazione scientifica della personalità, il giudice acquisisce la relativa documentazione e si avvale, se occorre, della consulenza dei tecnici del trattamento $\mathrm{e}$ in tal caso si effettua un accertamento sulla personalità. Si entra quindi nel procedimento di sorveglianza (art. 678 c.p.p. c.2) che consente di procedere a osservazione scientifica della personalità del condannato e che consente al giudice di richiedere la consulenza dei tecnici del trattamento e/o di esperti qualificati.

Il tribunale di sorveglianza nel caso in esame ritenne, come sopra ampiamente illustrato, non esauriente la documentazione riguardante l'osservazione scientifica della personalità della condannata, poiché il lavoro dell'équipe prevista dall'articolo $\quad 29 \quad$ comma 2 regolamento dell'esecuzione dell' ordinamento penitenziario, ha privilegiato l'aspetto trattamentale rispetto a quello dell'osservazione personologica. Anche in considerazione del fatto che la condannata ha sempre continuato a proclamarsi innocente, il collegio ha ritenuto necessario introdurre $e x$ officio (art. 666 comma 5 c.p.p., art. 185 disp. artt. c.p.p.) nel procedimento un nuovo mezzo di prova, vale a dire la perizia finalizzata ad accertare "il carattere e la personalità" del condannato e in genere "le qualità psichiche indipendenti da cause patologiche" (art. 220 comma 2 c.p.p.). Di conseguenza è stata disposta la consulenza, che può essere intesa pure come consulenza criminologica, e di cui qui di seguito si seguiranno le diverse fasi attraverso le quali si cercherà di mettere in evidenza se, mediante questa modalità di indagine, si è contribuito a realizzare un concreto programma per cui la pena si inserisce in un progetto rieducativo.

\section{Attraverso la consulenza tecnica d'ufficio.}

\subsection{Incarico.}

Il giorno 21 gennaio 2014, davanti al Tribunale di Sorveglianza di Bologna, presieduto dal Dott. Francesco Maisto, è stato affidato l'incarico di redigere consulenza tecnica d'ufficio nei riguardi di AMF, attualmente detenuta presso la Casa Circondariale di Bologna. Il Tribunale ha posto il seguente quesito: “dica il consulente tecnico d'ufficio, esaminati gli atti del presente procedimento e la documentazione pendente presso la Casa Circondariale di Bologna, esaminata AMF, esperiti gli opportuni accertamenti sull'attuale condizione fisica e psichica della condannata, anche avvalendosi della collaborazione di ausiliari, quali siano le condizioni della condannata, analizzi e descriva le condizioni della condannata. Dica altresì se sussistano condizioni di pericolosità sociale della condannata".

Dopo aver accettato l'incarico, il CTU ha indicato, per l'inizio delle operazioni peritali, il giorno 27 gennaio 2014 presso il suo studio, per l'esame degli atti. Ha inoltre chiesto il termine di giorni 70, a decorrere dal 27 gennaio 2014, per riferire con relazione scritta.

Il Tribunale di Sorveglianza ha concesso il sopra indicato termine e ha altresì autorizzato ad estrarre copia della documentazione processuale, ivi compresa quella esistente presso la Casa Circondariale di Bologna. Ha inoltre autorizzato 
ad accedere alla Casa Circondariale di Bologna per effettuare le necessarie operazioni. [...]

Per rispondere al quesito proposto, sono stati esaminati gli atti del procedimento e la documentazione acquisita. È stata trattenuta a colloquio AMF nei seguenti giorni: 4, 10, 17, 25 febbraio 2014, 6, 11, 17 e 27 marzo 2014. Ad eccezione dei giorni 4 e 17 febbraio, a tutti gli incontri è stato presente il consulente di parte della difesa ${ }^{5}$. Il giorno 17 febbraio sono stati somministrati a AMF una batteria di test. Il giorno 17 marzo, il CTU, presso il proprio studio, ha avuto un altro incontro con AMF e la sua famiglia composta dal marito e dai due figli. Il giorno 25 febbraio, durante il colloquio con AMF, sono stati acquisiti i saggi grafici, successivamente esaminati.

\subsection{Documentazione in atti.}

Nei riguardi di AMF, nella banca dati del casellario giudiziale risulta che il 27/04/2007 con sentenza della Corte di Assise di Appello di Torino divenuta irrevocabile il 21/05/2008, essa è stata condannata alla pena di reclusione di anni 16 per omicidio commesso il 30//01/2002 in Cogne, perché, colpendo alla testa il proprio figlio, ne cagionava la morte (art. 575 c.p., 577 comma 1 n.1 c.p.). Al momento in cui è stata disposta la consulenza tecnica d'ufficio (12/12/2013), AMF era detenuta presso la Casa Circondariale di Bologna in espiazione della pena detentiva residua di anni 13.

\footnotetext{
${ }^{5}$ Prof. Pietro Pietrini, Ordinario presso il Dipartimento di Patologia Chirurgica, Medica, Molecolare e dell'Area Critica, Università di Pisa, Settore scientifico disciplinare: Biochimica Clinica e Biologia Molecolare Clinica BIO/12. E' presidente del Consiglio Aggregato del Corso di Laurea in Scienze e Tecniche di Psicologia Clinica e della Salute e del Corso di Laurea Magistrale in Psicologia Clinica e della Salute.
}

Nella sentenza della prima Corte di Assise di Torino (2007) - esaminata - nella parte prima, si fa riferimento ai fatti riguardanti il ritrovamento, $i$ soccorsi e la dichiarazione di morte del figlio di AMF. [...]

Nella documentazione medico-legale e psichiatrica, sono stati presi in esame i risultati degli accertamenti redatti nel corso di numerose perizie psichiatriche e medico-legali effettuate nei riguardi di AMF. [...]

È stata poi studiata approfonditamente la documentazione psichiatrico-penitenziaria, estratta dalla copia della cartella clinica riguardante la detenzione di AMF presso la Casa Circondariale di Bologna. In tale contesto, è stata attentamente valutata l'evoluzione delle condizioni psichiche della condannata e le terapie effettuate per risolvere problematiche psicopatologiche che erano legate anche alla reazione alla detenzione e all'atteggiamento della condannata che si proclamava innocente e ingiustamente sottoposta a detenzione.

Inoltre, esiste agli atti una copiosa documentazione riguardante il trattamento penitenziario in cui vengono descritti gli interventi trattamentali effettuati, orientati al sostegno alla persona, che hanno riguardato principalmente il consentire alla stessa di svolgere un'attività lavorativa utile, secondo quelle che sono le esigenze, e le possibilità, del carcere, e la frequenza dei corsi di formazione professionale. Nell'ambito di questa relazione sono stati messi in evidenza i rapporti con le altre detenute e con il personale di polizia penitenziaria, definiti come privi di particolari problemi. All'inizio l'impatto con la realtà del carcere, con le storie personali di altre persone detenute, non ha mancato di 
evidenziare qualche difficoltà, ma in seguito ciò è stato superato anche in virtù di un apprezzabile sforzo prodotto da AMF nel farsi accettare. È inoltre segnalato che per quanto concerne la condotta, questa si presenta esente da rilievi di tipo disciplinare e che AMF incontra regolarmente in carcere i familiari, usufruisce infatti di 6 colloqui mensili che si svolgono, in considerazione dell'età dei figli, presso la ludoteca della sezione femminile; ella effettua altresì corrispondenza telefonica secondo quanto consentito.

AMF ha avuto anche l'opportunità di partecipare all'iniziativa denominata "Festa delle famiglie" (svoltasi con l'aiuto dei volontari operanti in istituto), caratterizzata da visite dei familiari di maggior durata.

Nel giugno del 2008 AMF, tramite i suoi legali, ha presentato un'istanza di ammissione all'art. 21 bis O.P. (“assistenza all'esterno di figli minori”), però non sussistevano le condizioni oggettive (poiché non è ancora avvenuta l'espiazione di almeno un terzo della pena) per fruire di tale istituto.

In data 07/10/2008 è stato emesso dal Presidente del Tribunale di Sorveglianza di Bologna decreto di inammissibilità riguardante le istanze di detenzione domiciliare (art. 47 quinquies O.P.) e differimento pena nelle forme della detenzione domiciliare (art. 47 ter co.1 O.P.) mancando per ciascuna delle misure alternative gli specifici presupposti di legge.

Infine risulta in questa relazione che la condannata è stata inserita presso il laboratorio esterno 'Gomito a Gomito' gestito da una cooperativa Sociale, mantenendo positivamente questo impegno di lavoro all'esterno.
L'esame di questi atti ha consentito di verificare l'impegno degli avvocati difensori nel proporre istanze e l'attenzione del tribunale di sorveglianza nel vagliarle. Inoltre, le direttive del trattamento si delineano in modo netto: assistenza psichiatrica-psicologica dall'inizio della carcerazione poi via via sostituita dalle attività trattamentali, orientate prevalentemente all'inserimento lavorativo e alla formazione professionale con concessione di svolgere successivamente un'attività all'esterno della struttura penitenziaria.

Gli elementi esposti sono da ritenere dati indispensabili per concorrere a costruire $i$ colloqui da cui possono scaturire elementi utili per la valutazione medico-psichiatrica $e$ in particolare per l'esame psichico.

2.3. Accertamenti medico-psichiatricopsicologici.

In questo settore, è stata raccolta la biografia clinica della condannata, indispensabile premessa all'esame psichico e agli ulteriori accertamenti. Attraverso la ricostruzione della biografia della condannata, si sono valutate le capacità mnemoniche, l'attenzione, l'ideazione, la capacità critica e di giudizio e l'affettività. Inoltre, si è fatto riferimento alle vicende esistenziali mettendo in rilievo i rapporti con la propria famiglia di origine, con il marito, con i figli e con tutte le persone con cui ha interagito, anche dopo che è stata imputata dell'omicidio del figlio e quindi condannata alla detenzione.

Nei diversi colloqui le tematiche affrontate suscitavano momenti drammaticamente significativi e quindi reazioni assai importanti da valutare per gli accertamenti in corso. Durante l'esame psichico si è potuto rilevare che la 
presentazione di sé fatta da $\mathrm{AMF}$ è tutta volta a trasmettere all'altro informazioni cercando di creare rappresentazioni positive. Da qui il complicato gioco dell'identità che si realizza attraverso l'ambiguità risultante dall' "essere" e dall' "avere". E' difficile separare nel soggetto il suo essere dal suo avere perché il legame tra questi due aspetti è talmente forte che ogni presentazione mobilita, in modo spesso indistinto, l'uno e l'altro aspetto del proprio Sé, tanto che, mettendo in discussione uno di questi aspetti, il rischio che corre è quello di rimettere in discussione pure l'altro. In rapporto a ciò, AMF tende frequentemente a porre gli avvenimenti in relazione alla propria persona in misura che oltrepassa ciò che è oggettivamente giustificabile. Pertanto, nel caso in esame, va sottolineata una pronunciata sensibilità per la coscienza del proprio valore, aspetti che orientano a valutare certi atteggiamenti come dettati dal perseguimento di un'autogratificazione, a volte svincolata da decisioni connotate da adeguata valutazione della situazione, facendo, in tal modo, di se stessa un oggetto di amore. In questa prospettiva, appare evidente il continuo bisogno di alimentare la propria autostima esibendo le proprie capacità e le proprie doti anche per i programmi futuri. Perciò si può dire che la sua esistenza, per quanto riguarda questi ultimi dodici anni, è connotata dalla spasmodica ricerca di quella che è stata definita "omeostasi narcisistica", una forma di equilibrio tra il perseguimento mai completamente raggiunto di una sensazione di benessere, distensione, quiete, da un lato, e il persistere di un senso di inferiorità, dall'altro, che si manifesta

$6 \quad$ Strzyz K., Narcisismo e socializzazione. Trasformazione sociale e il mutamento di dati caratteriali, Feltrinelli, Milano, 1981. nella continua esigenza di soddisfare l'immagine del sé. Il riferimento all'egocentrismo e al narcisismo consente di precisare che si tratta di caratteristiche della personalità che non sono da considerare sempre patologiche, poiché una quantità di amor proprio è auspicabile anche se, nel caso in esame, l'autostima appare accentuata per cui il soggetto diventa estremamente sensibile al modo con cui gli altri reagiscono nei suoi confronti.

Anche il desiderio di avere i figli accanto può diventare un tentativo inconscio di considerarli una parte del proprio Sé incompleto e frammentario. Con l'aiuto del bambino la madre corre il rischio di voler riempire le lacune della propria personalità e ciò significa che già in partenza, sempre a livello inconscio, ella va incontro al bambino esigendo qualcosa da lui.

Pure il ricordo della morte del figlio può essere inserito in questa dinamica. La morte del piccolo rappresenta poi un elemento significativo anche perché configura una seria rottura della continuità esistenziale. Riguardo alla morte del figlio, AMF ribadì spesso la propria innocenza e riferì che ormai da dodici anni ogni notte, ogni giorno pensa a quella mattina. [...]

La rievocazione di tali eventi, emotivamente molto partecipata, sfociava in crisi di pianto e in manifestazioni ansiose. [...] Ci si trova di fronte ad un travaglio psichico prolungato, sicuramente influenzato dagli eventi esterno-ambientali, che ha prodotto uno sradicamento e una perdita dell'ambiente consuetudinario provocando uno stato d'animo informato ad un'evidente tristezza astioso-lamentosa con notevole ansia.

In queste vicende giudiziario-esistenziali, ci si può trovare di fronte alle idee prevalenti o dominanti, 
definite "come un contenuto di coscienza (rappresentazione, pensiero, impulso) che ha caratteri iterativi e, talvolta, pervasivi ma senza il carattere dell'estraneità in quanto essa [idea prevalente o dominante] è comprensibilmente collegata ad una particolare condizione emotiva. Il carattere egosintonico dell'idea dominante è il carattere differenziale più importante nei confronti dell'idea ossessiva. Il contenuto di coscienza, anche se iterativo, pervasivo, al punto da interferire con il normale svolgersi delle attività ideative e comportamentali, non viene mai percepito come estraneo, assurdo e incomprensibile come avviene con le idee ossessive. Esso viene invece accettato più che subito e in alcuni casi in un certo senso voluto e volontariamente rinforzato. Esempi classici in questo senso possono essere rappresentati dall'ideazione dominante che segue in genere la perdita di una persona amata o quella che accompagna una potente condizione di attaccamento come quella rappresentata dall'innamoramento. La partecipazione affettiva all'idea dominante è intensa, congrua e considerata soggettivamente come adeguata e proporzionata, anche se può obiettivamente apparire come eccessiva. Questo carattere egosintonico e affettivamente condizionato giustifica il fatto che non è in genere presente né resistenza né psichismo di difesa anche quando l'idea dominante tende ad imporsi e sovrapporsi alla normale fluidità e plasticità dell'attività mentale. Ciò giustifica il fatto che l'idea dominante non viene in genere considerata come patologica ma piuttosto come una motivata e giustificata conseguenza di uno stato emozionale, spesso indotto da eventi stressanti esterni",7.

La presenza di idee dominanti si collega strettamente alle intense manifestazioni depressive con espressione di ansia che sono correlate alle vicende che hanno caratterizzato la vita di AMF in questi dodici anni, anche se nel corso dei colloqui, ha ricordato la vita felice a Cogne. [...]

Durante l'incontro con AMF, il marito e i loro due figli si è potuto constatare che $\mathrm{AMF}$ aveva un ottimo rapporto con $\mathrm{i}$ propri familiari che si estrinsecava in atteggiamenti affettuosi e di autentica confidenza. [...] Durante i colloqui, $\mathrm{AMF}$ ha affrontato con atteggiamento sincero la sua vita nell'ambiente carcerario ponendo in rilievo le difficoltà iniziali e il successivo adattamento, seppur faticoso, alle diverse attività "rieducative". Inoltre, è stato ricordato con soddisfazione e con piacere il più recente impegno per il lavoro all'esterno, in ambito sartoriale, presso il Laboratorio "Gomito a gomito", gestito dalla cooperativa sociale "Siamo qua".

Un aspetto di ulteriore serenità, sollievo e fiduciosa aspettativa per il futuro sono i permessi che ogni quindici giorni le consentono di rientrare in famiglia, procurandole una felice e totale immersione nel proprio ambiente familiare, collocato in un contesto naturale particolarmente apprezzato da AMF. Circa i legami con i familiari, ha ribadito spesso che tutti i colloqui che le erano concessi durante la sua detenzione li riservava ai figli e al marito, cercando in tal modo di conservare immutato il profondo legame che li unisce.

\footnotetext{
7 Pancheri P., Brugnoli R., Marconi P.L., Carilli L., "Pensiero", in Pancheri P., Cassano G.B. (coordinato da), Trattato italiano di psichiatria, vol. I, Masson, Milano, 1999, pp.547-548.
} 
In conclusione, nei diversi colloqui con AMF, oltre alle idee dominanti ed ai tratti di personalità definiti come egocentrici e narcisistici, emergono anche manifestazioni depressive ed ansiose estrinsecantesi con pianto e con irrequietezza tipica dell'ansia. In questa prospettiva, la depressione di AMF, da considerarsi come reattiva, trova il momento determinante nei seguenti avvenimenti: morte-uccisione del figlio, vicende giudiziarie, condanna e ingiusta detenzione. Questa serie di avvenimenti è di per sé valida a produrre un sovvertimento della configurazione totale delle disposizioni psichiche nel senso di una coartazione dolorosa della sfera timica polarizzata ancora sugli eventi che hanno contrassegnato l'esistenza di AMF negli ultimi dodici anni.

I dati evidenziati dall'esame psichico trovano conferma nelle valutazioni psicodiagnostiche emerse dai seguenti Test: Rorschach, MMPI-2, Bender Test, Figura umana e analisi della personalità grafica. In particolare, il test di Rorschach evidenzia che la dotazione intellettiva di base appare media, al momento sostanzialmente espressa senza particolari forme di inibizione. A questo proposito, il soggetto conferma una necessità di coinvolgimento affettivo come elemento attivatore, che abbassa leggermente gli schemi difensivi, favorendo un certo dinamismo psichico e un pensiero più fluido e meno rigido. $\grave{E}$ possibile infatti che quando il soggetto si trova da solo in contatto con se stesso si attivino aspetti soprattutto depressivi che favoriscono un irrigidimento dei nessi associativi. Inoltre, per quanto concerne l'intelligenza qualitativa, il tipo di pensiero appare al momento prevalentemente portato alla deduzione e ad un approccio fondato appunto su meccanismi più riproduttivi che produttivi, nonostante non manchino spunti di originalità.

L'esame di realtà appare adeguato, in assenza di risposte che segnalino distorsioni percettive sia di matrice psicotica che organica. Sul piano affettivo emergono bisogni di contatto ed in parte un tentativo di allontanamento dal mondo interno per gli aspetti depressivi di fondo. I meccanismi difensivi appaiono evoluti ed orientati alla rimozione non patologica. Si osserva una certa attenzione al corpo, bisogni narcisistici, ma soprattutto tendenze regressive, viste le molte figure caratterizzate come "cuccioli", "elefantini", "uccellini", che poi si confermeranno anche in altri test. [...]

Il test MMPI-2 ha manifestato un marcato atteggiamento difensivo nei confronti della prova, cercando di minimizzare e negare aspetti della personalità considerati sfavorevoli ai fini della valutazione, fornendo così un'immagine idealizzata e irrealistica sul piano del conformismo sociale.

Al Bender test emergono segnali che sono compatibili con una forma di egocentrismo e bisogno di centralità affettiva di stampo narcisistico, che rendono difficoltoso il processo di anticipazione e di pianificazione.

La figura umana si esprime attraverso disegni in cui le due figure complete sono caratterizzate da un'età sensibilmente inferiore a quella del soggetto, confermando aspetti regressivi, sicuramente in massima parte addebitabili allo stato di detenzione, ma anche alla polarizzazione del pensiero sui figli e conseguente atteggiamento materno nei confronti del sesso opposto. Anche in 
questo senso potrebbe essere letto il fatto che la prima figura è maschile e non femminile. [...]

Non emergono in assoluto tratti psicopatologici degni di rilevanza clinica, nonostante si osservino aspetti regressivi riguardo all'espressione del ruolo femminile, appunto in probabile relazione con la detenzione.

Si è utilizzata poi l'indagine grafologica per interpretare la sindrome grafica nel suo insieme cercando di dare una giusta collocazione ai vari segni senza trascurare l'analisi dei rapporti esistenti tra questi ultimi al fine di meglio comprendere la personalità dello scrivente.

AMF all'esame grafologico, per quanto riguarda temperamento e carattere, appare persona tendenzialmente buona di animo e comprensiva, ma preoccupata del giudizio degli altri per cui assume atteggiamenti coscientemente controllati. Presenta poi un orgoglio frustrato, quasi rassegnato a non pretendere e a non chiedere. Persiste in lei un'ambivalenza tra spinta all'orgoglio e la cedevolezza nella quale deve ripiegare per il suo senso interiore di debolezza che la fa essere strutturalmente remissiva, assumendo atteggiamenti improntati alla riservatezza e alle modalità guardinghe, come chi teme di sbagliare. Esteriormente può apparire forte, perché in gran parte riesce a mascherare le sue debolezze sotto la sostenutezza più apparente che reale, ma interiormente fragile, sia per le apprensioni improvvise, anche se di breve durata, sia per le sospensioni dell'animo che la portano verso stati di tristezza.

Tende a compensare le insoddisfazioni con il meccanismo della fantasia che la porta a sognare ad occhi aperti situazioni diverse. E' orientata positivamente nei propositi e nell'azione, ma subentrano in lei oscillazioni dello stato d'animo e del tono umorale, tra momenti di sconforto interiore e momenti di entusiasmo e di ottimismo. Per quanto riguarda l'intelligenza, le sue abilità si manifestano nei confronti delle situazioni che esigono attenzione in tutti $\mathrm{i}$ particolari $\mathrm{e}$ compitezza di esecuzione. Non tutto ciò che entra nel campo di coscienza e non tutto ciò che entra nella sua comprensione viene adeguatamente accettato e filtrato al vaglio del giudizio, in quanto l'animo, non sempre sereno, ne condiziona le valutazioni.

Nei colloqui si è manifestato "il parlare inteso come sfogo" quasi come si verifica per il pianto in reazione al dolore, particolarmente quando l'attenzione era rivolta all'esaminata-condannata che esprimeva le sue emozioni riattualizzando l'episodio traumatico. Con l'intervento dei suoi interlocutori (CTU e CT di parte), AMF prendeva coscienza della sua situazione soprattutto per quanto riguardava la finalità dell'indagine: farsi conoscere, conoscere per cui si potesse decidere. Il colloquio quindi, come base dell'esame psichico, nella perizia criminologica, allorchè si realizza in una buona relazione, oltre a fornire informazioni attendibili, assume un significato benefico e quindi positivo con valenze che si ripercuotono anche a livello psico-terapeutico.

Attraverso l'abilità (intesa come requisito professionale) nell'osservare e nel valutare la condannata, come persona, il CTU e il CT di parte ritennero di esserle stati, in qualche modo, pure utili e benefici, anche se la raccolta degli elementi necessari era strettamente legata al progetto finale: fornire risposte attendibili $e$ obiettive ai quesiti posti che dovevano poi essere inseriti nel più ampio progetto rieducativo. 
Questi contributi sono il presupposto da utilizzare nelle considerazioni e valutazioni del caso e rappresentano quelle anticipazioni, ragionate $e$ critiche, alla conclusione della relazione di consulenza.

\subsection{Analisi dei dati e valutazione della storia della} persona come punto di riferimento nel progetto rieducativo.

Sulla scorta della documentazione acquisita e sulla base di quanto osservato nel corso degli accertamenti effettuati, è opportuno segnalare che situazioni ed avvenimenti della vita di AMF possono aver influito sulle strutture di personalità e soprattutto sull'affettività e sull'emotività per cui si è fatto riferimento al concetto di reazione ad eventi. E' noto nella letteratura psichiatrica che in risposta a fatti o a più fattori stressanti identificabili si possono sviluppare sintomi o comportamenti clinicamente significativi. I fattori stressanti che si possono individuare nella vita di AMF sono: la morte-uccisione del figlio, la vicenda giudiziaria e la condanna ritenuta ingiusta, la carcerazione, causa di sofferenza, e la preoccupazione per individuare il vero colpevole dell'omicidio.

A ciò devono aggiungersi i conflitti con personaggi da cui ritiene di essere stata danneggiata, quelli collegati all'ingiusto trattamento subito dalla presunta "persecuzione mediatica" e alcuni dissapori con persone a lei vicine. Perciò, nei riguardi di AMF può essersi instaurata quella situazione che è considerata "una risposta psicologica ad uno o più fattori stressanti identificabili che conducono allo sviluppo di sintomi emotivi o comportamentali clinicamente significativi" ${ }^{8}$.

I sintomi possono persistere per un periodo prolungato allorché si manifestano in risposta ad un fattore stressante cronico e sono caratterizzati da condizioni in cui predominano ansia e umore depresso: tendenza al pianto con atteggiamento di rivendicazione per le ingiustizie e i torti subiti, irrequietezza e costante preoccupazione legate soprattutto al futuro.

Nell'ambito della relazione di perizia, si è fatto ricorso ad uno schema multiassiale che comporta la valutazione su diversi assi di informazioni psico-medico-sociali, come è proposto nel DSMIV-TR, apportandovi le modifiche ritenute opportune per il caso in esame dato che quando i criteri, le categorie e le descrizioni vengono utilizzati a fini forensi si corrono molti rischi se si resta aderenti a schemi prefissati. Tali rischi vengono senz'altro neutralizzati se ci si attiene strettamente alla biografia della persona in esame e ai dati obiettivi emersi dalla documentazione e dalle indagini clinico-psichiatriche e psicodiagnostiche. Di conseguenza, prendere in esame contestualmente i risultati del colloquio, di diversi reattivi mentali e la storia di vita è un approccio che può produrre validi risultati in ambito criminologico-psichiatrico consentendo anche di ricorrere alla valutazione multiassiale.

Nella sintesi diagnostica, non va trascurato che lo spazio vitale attuale di AMF è molto condizionato dal futuro psicologico: infatti, negli studi sulla sofferenza in carcere si è constatata una sicura relazione tra il grado della sofferenza e certi fattori collegati al futuro $\mathrm{o}$ al passato. $\mathrm{La}$

\footnotetext{
8 American Psychiatric Association, DSM -IV -TR, Manuale diagnostico e statistico dei disturbi mentali, Masson, Milano, 2003, p. 723.
} 
convinzione di una sentenza ingiusta e la speranza per una possibile liberazione sono elementi interattivi che provocano ansia e sofferenza per cui si è ritenuto necessario che $\mathrm{AMF}$, per poter accedere ad una misura alternativa alla detenzione, dovesse essere adeguatamente supportata attraverso un percorso di sostegno psicoterapeutico. AMF è stata adeguatamente trattata nel primo periodo di detenzione, al fine di neutralizzare ansia e depressione, conseguendo positivi risultati. E' stata inoltre ben inserita nelle attività trattamentali (corsi di formazione e lavoro all'interno e all'esterno), ma, pur avendo avuto un adeguato supporto psicoterapeutico mediante colloqui di sostegno, necessita, per affrontare adeguatamente l'attuale situazione, di ulteriori percorsi di aiuto e di sostegno.

Il passaggio dal carcere all'alternativa alla detenzione pone un soggetto di fronte a stimoli percettivi più complessi e numerosi provenienti dalla mutata realtà circostante per cui un individuo può non essere in grado di utilizzare pienamente i processi cognitivi come strumento per il mantenimento della sicurezza nei rapporti Io-mondo. [...]

D'altronde anche i risultati delle numerose consulenze e perizie, pur evidenziando ampie diversità valutative per quanto concerne le caratteristiche minori della personalità di AMF, escludevano la presenza di conclamate e maggiori patologie psichiatriche. [...]

Perciò, nel caso in esame, tenendo conto delle caratteristiche di personalità e della situazione psicologica attuale, occorre orientare il trattamento verso un'adeguata psicoterapia di supporto, necessario passaggio per poter proficuamente inserire la condannata nel proprio ambito familiare.

Questi ultimi paragrafi rappresentano la sintesi con cui si è conclusa la relazione di consulenza tecnica di ufficio.

\section{Approfondire per decidere.}

Il 29 aprile 2014 il Tribunale di Sorveglianza', all'esito dell'esame della relazione di consulenza tecnica d'ufficio e riservato l'esame della relazione del consulente tecnico di parte, dopo aver messo in evidenza che si verte unicamente in tema di eventuale concessione della detenzione domiciliare speciale ex art. 47 quinquies ordinamento penitenziario, ritenuta la necessità di sentire a chiarimenti il CTU su specifici contenuti della relazione suddetta, indicava i seguenti temi di approfondimento peritali:

1. Durata, numero di ore ed andamento dei permessi premio del beneficiario ex art. 21 ordinamento penitenziario.

2. Discussione della documentazione acquisita ed oggetto di ordinanze del Tribunale per i minorenni di Bologna in tema di potestà genitoriale di AMF.

3. Contenuti e limiti della Ctu del 2008 dell'Ufficio di Sorveglianza di Bologna.

4. Aspetti logistici e relazionali di eventuale collocazione domiciliare in collaborazione con l'Uepe (Ufficio di Esecuzione Penale Esterna) di Bologna.

5. Se per "condizioni di pericolosità sociale" si intenda il pericolo di recidiva oppure la generica pericolosità sociale.

\footnotetext{
${ }^{9}$ Il Tribunale di Sorveglianza, riunito in Camera di Consiglio nelle persone di: Dott. Francesco Maisto Presidente, Dott. Sabrina Bosi - Magistrato di Sorveglianza est, Dott. Cinzia Bertuccioli - esperta,
} 
6. Precisazione sulla eventuale relazione temporale tra psicoterapia di supporto ed eventuale misura alternativa.

In questa fase la progett-azione e la costruzione della relazione di consulenza tecnica d'ufficio deve acquisire nuovi dati ed effettuare particolari approfondimenti che fanno specifico riferimento a documentazione esistente presso strutture dell'amministrazione della giustizia per una più analitica valutazione del comportamento futuro $e$ quindi della sussistenza della pericolosità sociale, tenendo conto delle eventuali modalità operative relative all'impostazione di una psicoterapia di supporto.

\subsection{Supplemento di indagine.}

$\mathrm{Da}$ ciò è scaturito un approfondimento $\mathrm{e}$ supplemento di indagine che si è sviluppato attraverso le attività e le indagini qui di seguito precisate:

1. raccolta presso il Tribunale di Sorveglianza di Bologna della documentazione relativa ai permessi ottenuti da AMF dal settembre 2010 al marzo 2014;

2. acquisizione della documentazione oggetto di ordinanze in tema di potestà genitoriale di AMF presso il Tribunale per i Minorenni di Bologna;

3. analisi della relazione di CTU del 2008 dell'Ufficio di Sorveglianza di Bologna al fine di valutarne contenuti e limiti;

4. esame della documentazione pervenuta al Tribunale di Sorveglianza e redatta dal un operatore esperto dell'UEPE, riguardante aspetti logistici e relazionali, per eventuale collocazione domiciliare di AMF;

Dott. Stefano Rambelli - esperto, ha disposto una integrazione della perizia.
5. esame della relazione di consulenza tecnica già depositata, della documentazione ad essa allegata e dell'ulteriore documentazione acquisita ai fini di verificare se per "condizioni di pericolosità sociale" si intenda il pericolo di recidiva oppure la generica pericolosità sociale.

Nello strutturarsi della relazione di consulenza tecnica di approfondimento, in primo luogo sono state redatte alcune precisazioni relative alle considerazioni del consulente di parte della difesa e si è quindi preso in esame l'andamento dei permessi. In particolare si è rilevato che AMF ha ottenuto, dal 31.08.2010 al 22.04.2014, diversi permessi premio, che consistono in circa ore $940 \mathrm{e}$ minuti 50, corrispondenti a circa giorni $39 . \mathrm{La}$ detenuta si è ogni volta regolarmente presentata in Istituto, dopo aver consumato i permessi senza particolare rilievi e con fruttuose esperienze. Eccetto i primi due permessi che avevano particolari finalità (partecipare al funerale del suocero e assistere alla malattia del marito), tutti i permessi successivi sono stati finalizzati a coltivare gli affetti familiari.

Per quanto riguarda contenuti e limiti della C.T.U. 2008 dell'Ufficio di Sorveglianza di Bologna, si è sottolineato che tale consulenza risale al secondo semestre 2008, periodo in cui AMF era da poco sottoposta a carcerazione, e pertanto sarebbe stato ancora più opportuno approfondire il percorso esistenziale di AMF non tanto avvalendosi di particolari metodiche psicodiagnostiche che possono apparire profondamente influenzate dai colloqui, quanto piuttosto facendo un maggior riferimento alla documentazione psicosociale esistente e proveniente da diversi servizi.

Se si tiene conto delle metodiche usate e del periodo in cui questa CTU si è svolta, si può 
sostenere che questo accertamento è ormai superato e non risponde alle esigenze richieste dalle attuali indagini.

Relativamente agli aspetti logistici e relazionali contenuti nella relazione UEPE, viene messo bene in evidenza la profondità del legame con i figli da parte di AMF, la straordinaria sensibilità del marito e la disponibilità ad ottenere un supporto da parte dei servizi necessario per il delicato passaggio che entrambi i figli vivono nella fase adolescenziale e quindi un passato di impegno dei coniugi nella prospettiva di vita in paese in cui ognuno potrà assumere ben definiti atteggiamenti e adeguate condotte così che si realizzi un equilibrato sistema familiare che esige appunto la consapevolezza dei diversi ruoli.

In relazione alla pericolosità sociale, si legge che nella sentenza della Prima Corte d'Appello d'Assise di Torino (2007), si è posto il quesito della causale del figlicidio commesso da AMF e si è precisato che "la causa scatenante dell'azione delittuosa risiede probabilmente in un conflitto interiore, ed è da ravvisarsi in quella stessa causa che ha provocato l'insorgere della sintomatologia ansiosa di AMF". Inoltre in questa sentenza si precisa ancora che "l'imputata, dato il suo assetto difensivo, pregno di negazione e di idealizzazione [...] celava certamente a se stessa l'origine della sua ansia patologica facendo ricorso al mascheramento [...] E' plausibile che 'il polo nascosto' risiedesse in una forte preoccupazione di AMF per il figlio, che soffriva di intolleranze alimentari e di temporanei ritardi nell'accrescimento, come dimostrano i documenti clinici che riguardano i controlli e i brevi ricoveri del bambino: elementi di fatto che, secondo la Corte, avevano dato motivo (in famiglia) a definirlo, sia pure in tono affettuoso e con intento apparentemente scherzoso, 'nanetto', 'con le gambe ad X', o 'con le gambe secche' con espressioni che lo designavano, in effetti, come un soggetto che creava inquietudine, e forse delusione (almeno in AMF), nonostante che l'imputata stessa, secondo un meccanismo di idealizzazione a lei tipico, ne abbia, anche in giudizio, esaltato entusiasticamente la bellezza e l'indole gioiosa e vivace, oltre che il valido legame fraterno con il fratello".

Dalla citata sentenza appare inoltre importante segnalare quanto qui di seguito si riporta:

"A ciò va collegata, per comprendere l'incidenza dei 'problemini' (recentissimi) del figlio sulla tendenza alla teatralità e alla drammatizzazione che contraddistinguono il funzionamento [...] dell'imputata, l'abitudine di AMF stessa di rifugiarsi al paese natale, presso la vasta e Patriarcale famiglia d'origine, in funzione di terapia delle sue fasi critiche, dipendenti anche dall'isolamento che caratterizzava molta parte della sua giornata in Cogne, in un'abitazione lontana dal centro abitato, e in assenza del marito, a causa della sua attività di lavoro nei dintorni di Aosta.

Il tema del conflitto familiare non rivelato - anzi, dissimulato con il ricorso all'idealizzazione e all'autoreferenzialità tipiche di AMF, che vede se stessa al centro delle 'cose buone', senza concessione di riconoscimenti positivi al mondo esterno 'non familiare' e non 'accudente' rispetto a lei - intrecciato con $\mathrm{i}$ malesseri ansiosi e conversivi dell'imputata, particolarmente seri nelle ore serali del 29 e al mattino del 30 gennaio,

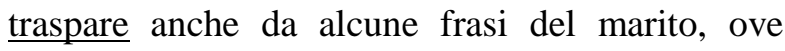
questo dichiara di essersi sentito inquieto quel 
mattino, nel lasciare la moglie e i bambini, per aver previsto che AMF lo avrebbe richiamato, e che egli si sarebbe trovato nella difficoltà di parlarle in una probabile situazione di urgenza (che paventava accadesse), perché il suo cellulare era scarico. [...] Va ricordato che AMF disse di aver presagio che il figlio sarebbe morto e che forse 'aveva una menomazione' (vi è da chiedersi come mai le inquietudini e i presagi del male futuro fossero incentrate, da una parte, solo sul figlio, per una sua probabile debolezza, che lo avrebbe facilmente esposto agli attacchi di un'inconoscibile sorte funesta ['me la sentivo che sarebbe morto', 'facciamo un altro figlio'], e, dall'altra, per quanto attiene alla condizione psicologica di allarme del marito, il mattino del 30 gennaio, sulla capacità di AMF di ristabilirsi dalla crisi molto seria che l'aveva fatta soffrire per molte ore, nella sera-notte e al mattino).

La sindrome d'ansia patologica di AMF perdurò, come si è detto, dopo la partenza del marito, che se ne era andato all'ora consueta, dopo una momentanea incertezza 'se andare o restare' (risolta con l'uscita da casa, su invito della moglie) e probabilmente aumentò di intensità, quando l'imputata si rese conto della complicazione derivante l'inatteso risveglio del figlio, che protraeva $\mathrm{i}$ tempi della sua personale preparazione, al fine di raggiungere l'altro figlio in modo di riuscire a farlo salire sullo scuolabus.

Il figlio si era risvegliato e piangeva, avrebbe (forse) voluto uscire con il fratello, o non separarsi dalla mamma, così che $\mathrm{AMF}$, che si trovava a dovere fronteggiare un bambino inquieto e riottoso, capì che il figlio creava serie difficoltà all'organizzazione che lei aveva disegnato per quella mattina. L'organizzazione delineata da una persona 'puntuale' come AMF [...], minacciava di restare bloccata, per 'colpa' del figlio, che si poneva in ostinato contrasto con il piano della madre 'accudente'.

AMF, molto stanca per aver dormito poco e male durante la notte, a causa dell'acuzie della crisi d'ansia e dei connessi fenomeni conversivi, desiderava (il 30 gennaio) di lasciare a casa il figlio minore, per rimettersi a riposo, dopo aver accompagnato il figlio maggiore, e stare, infine, vicina al più piccolo, trascorrendo tranquillamente il mattino in casa con lui (pare da escludere, infatti, che, nelle descritte condizioni di malessere psico-fisico, AMF intendesse, una volta rientrata a casa, rimettersi in movimento, in una sorta di emergenza, per preparare il figlio per la scuola materna, in tempo utile al 'terzo giro dello scuolabus' - giro destinato al trasporto dei bimbi ).

Il figlio si mise non solo a piangere, ma si alzò e fu trasferito dalla mamma nel 'lettone', dandogli a credere $[\ldots]$ che la mamma non sarebbe affatto uscita di casa.

La 'ribellione' del figlio diede avvio alla reazione violenta dell'imputata, ansiosa, sofferente, stanca ed arrabbiata, in presenza di un discontrollo, favorito dallo stato ansioso e dall'indicato fattore scatenante, di natura emotigena ). [...]

Nel rilevare quanto precede, la Corte, in effetti, non si discosta dall'indicazione probabilistica data, in proposito, dal Giudice di primo grado, vale a dire, che AMF, trovandosi al cospetto di un figlio vivacemente 'opponente' (l'imputata stessa [giova aggiungere] ha dichiarato che il figlio aveva un 'carattere' più forte di quello del docile fratello maggiore), in preda ancora all'acuzie della sindrome ansiosa, $[\ldots]$ abbia agito contro il 
bambino a scopo punitivo, 'lasciandosi poi $\underline{\text { trasportare da incontrollata ira sino al selvaggio }}$ omicidio commesso' [...]".

In questa interpretazione del movente del delitto attribuito ad AMF, emergono circostanze e fatti che pongono in evidenza come il comportamento sia funzione della persona $(\mathrm{P})$ e dell'ambiente (A), per cui è legittimo ricorrere alla psicologia topologica di Kurt Lewin ${ }^{10}$, secondo cui ogni atto che una persona compie è in rapporto a particolari condizioni che sarebbero da ricercare appunto in parte nello stato della persona al momento considerato $\mathrm{e}$ in parte nelle caratteristiche dell'ambiente psicologico in cui si trova. Il comportamento di AMF può essere quindi considerato come funzione dell'ambiente e della persona secondo l'espressiva formula lewiniana: $\mathrm{C}=\mathrm{f}$ (P.A), che consente di approfondire la condotta di una persona e di svolgere considerazioni pure in prospettive prognostiche.

Al momento del fatto per cui è stata condannata, AMF si trovava, secondo la citata sentenza, in una particolare condizione psicologica ed aveva di fronte una situazione ambientale caratteristica. Dopo poco più di dodici anni dal fatto (30.01.2002), è evidente che una tale costellazione di eventi non è più riscontrabile e ciò consente di sostenere che non vi sia il rischio che si ripeta il figlicidio come descritto nella citata sentenza della Corte d'Asside d'Appello di Torino. Pertanto, non si può sostenere la sussistenza di una pericolosità

10 Balloni A., Criminologia in prospettiva, Clueb, Bologna, 1983; Balloni A., Bisi R., Sette R., Manuale di Criminologia - Volume I - Le Teorie, Clueb, Bologna, 2013; Balloni A., Bisi R., Sette R., Manuale di Criminologia - Volume II - Criminalità, Controllo, Sicurezza, Clueb, Bologna, 2013; Balloni A., "La teoria del campo di Kurt Lewin e le sue applicazioni in criminologia", Rivista di Criminologia, Vittimologia e Sicurezza, Vol. III, N.3 - Vol. IV - N.1, Settembre sociale specifica. Per condizione di pericolosità sociale deve intendersi, in relazione agli accertamenti effettuati, la generica pericolosità sociale, che si fonda sul presupposto che una persona abbia commesso un fatto previsto dalla legge come reato e che possa commettere nuovi fatti preveduti dalla legge come reati. Perciò, si può ribadire che quando si formulano previsioni sulla pericolosità sociale, per essere attendibili, occorre tener conto di quelle variabili dalle quali dipende il comportamento, cioè la persona e l'ambiente.

In questa prospettiva si può quindi ancora fare riferimento ai concetti lewiniani secondo cui il comportamento di una persona è funzione delle caratteristiche della persona medesima e dell'ambiente.

In effetti, la "persona" AMF è stata descritta con particolari caratteristiche di personalità. Nelle condizioni che vengono approfonditamente descritte nella relazione di perizia, ella deve affrontare l'ambiente di vita con esigenze lavorative, con impegni educativi nei riguardi dei figli e con realtà ambientali difficilmente prevedibili nella loro complessità. Nell'interazione individuo-ambiente è evidente che, tenendo conto della storia di vita di AMF, la medesima correrà rischi di incontrare difficoltà, frustrazioni, contrasti ambientali e altri avvenimenti che potranno incidere sul suo comportamento. [...]

Perciò è stata proposta una psicoterapia di sostegno al fine di rimuovere i disturbi esistenti e di cambiare i modelli di comportamento per promuovere uno sviluppo e una crescita positiva della personalità e per disporre di un ulteriore

2009 - Aprile 2010, pp. 171-186. 
strumento idoneo a neutralizzare i rischi di future condotte devianti e/o antigiuridiche.

Dalla documentazione acquisita presso il Tribunale per i Minorenni di Bologna, approfonditamente esaminata, con particolare riguardo all'ordinanza del Tribunale per i Minorenni di Bologna, in tema di potestà genitoriale di AMF, risulta che la medesima è sempre stata giudicata positivamente per quanto riguarda le sue condotte relative alla capacità genitoriale. È stata poi sottolineata la preoccupazione manifestata da AMF soprattutto legata alla sua assenza dal nucleo familiare che si poteva ripercuotere negativamente sulla crescita del figlio minore. Al marito di AMF sono sempre stati attribuiti comportamenti congrui $\mathrm{e}$ responsabili per quanto riguarda la sua disponibilità educativa e relativamente al suo impegno lavorativo dopo i fatti che hanno portato alla morte del figlio. I minori hanno manifestato difficoltà ad adattarsi dopo il decesso del fratello e dopo il trasferimento da Cogne [...]. Occorre precisare che i minori sono sempre stati sottoposti ad attenzione da parte dei servizi sociali e territoriali, in ambito psicologico, pedagogico e neuropsichiatrico infantile, tanto che i trattamenti e l'assistenza effettuati hanno avuto influenze positive sul loro sviluppo psicofisico.

E' da segnalare come dalla dinamica tra uffici giudiziari (Procura della Repubblica e Tribunale per i Minorenni) sia emersa la necessità di un'importante attività di sostegno psicosociale nei confronti di AMF che, attraverso la rete dei servizi, potrà proiettarsi anche nel futuro.

Il tema centrale del supplemento di indagine è senz'altro legato alle problematiche riguardanti la psicoterapia di supporto e le misure alternative alla detenzione. Nell'ambito delle alternative alla detenzione, si possono sviluppare sul piano rieducativo e quindi del trattamento tre diversi momenti che riguardano la crescita psicologica dell'individuo, un buon adattamento al suo ambiente e una maggiore capacità di soluzione dei problemi che la vita comporta. Innanzitutto occorre far leva sul fatto che il candidato alla psicoterapia manifesti la sua consapevolezza di aver bisogno di sostegno e di aiuto. Perciò è necessario, come è il caso di AMF, che nel lavoro psicoterapeutico il soggetto venga attivamente coinvolto, incoraggiandolo a manifestare le sue emozioni, soprattutto quelle legate all'ansia, ai sentimenti di colpa e all'aggressività. Per sviluppare questo approccio, si propongono tre momenti:

1) securizzazione;

2) valorizzazione;

3) affrancamento.

Per quanto riguarda la securizzazione, questa fase è la premessa per ogni eventuale ulteriore progresso: si tratta di dare sicurezza ad un soggetto che esprime ansietà, che presenta manifestazioni depressive reattive con idee dominanti legate al dramma giudiziario vissuto. In una tal prospettiva, si fa riferimento alla cosiddetta "terapia centrata sul cliente", elaborata da Rogers, quale strumento aperto e stimolante per l'interpretazione della realtà ${ }^{11}$. Perciò il terapeuta dovrebbe accettare ogni aspetto dell'esperienza di chi è sottoposto alla psicoterapia, vivendo verso di lui una condizione positiva senza esprimere valutazioni o giudizi. In effetti, "più l'individuo è capito ed accettato 
profondamente, più tende a lasciar cadere le false 'facciate' con cui ha affrontato la vita e più si muove in una direzione positiva di miglioramento" ${ }^{, 2}$. La direzione verso cui il sottoposto a terapia, per acquisire sicurezza e liberarsi dei falsi concetti di sé, deve collegarsi al principio della non direttività per cui Rogers, in genere, consiglia di limitarsi a fornire al cliente risposte che possono rendere più chiaro il significato dei suoi sentimenti. In una tal ottica, occorre far leva su quanto ha spinto il soggetto verso la psicoterapia mettendo in risalto la sua consapevolezza di aver bisogno di sostegno e di aiuto.

In una fase successiva, allorché si tende alla valorizzazione del soggetto, vengono messe in atto nei suoi confronti atteggiamenti favorevoli allo sviluppo di sentimenti positivi per sostenere un piano di vita rinnovato in sintonia sempre maggiore con i cambiamenti avvenuti nella personalità del sottoposto alla psicoterapia. In tal modo dovrebbe modificarsi il concetto di sé che è uno dei punti centrali della relazione di aiuto: nel corso della psicoterapia un concetto di sé rigido e impreciso, dovrebbe essere abbandonato e il soggetto dovrebbe essere facilitato ad accettare anche i valori provenienti dal mondo esterno, sforzandosi di conservare e accentuare in molteplici modi l'affetto di persone significative (marito e figli, ad esempio), abbandonando esperienze non compatibili con questi atteggiamenti. La fine della terapia, quando si realizzerà, costituisce appunto il momento definito dell'affrancamento, in cui il soggetto saprà gestire la complessità dei propri sentimenti attraverso una

11 Rogers C.R., La terapia-centrata-sul -cliente, G. Martinelli Editore, Firenze, 1970.

${ }^{12}$ Ibidem, p. 45. sempre maggiore autonomia, autostima e sicurezza.

Si è fatto riferimento, come esempio applicabile, a questo tipo di psicoterapia, alla cosiddetta terapia centrata sul cliente, perché, rispetto ad altre tradizionali psicoterapie, si differenzia in un punto fondamentale: non si occupa esclusivamente del "malato", ma anche di situazioni di "normalità".

Nella prospettiva della psicologia della normalità, occorre ricordare che gli esseri umani tendono spontaneamente a mantenere e a rafforzare $\mathrm{i}$ propri equilibri psichici, per cui più che curare una malattia come è nel caso in esame, occorre incoraggiare strategie e prassi finalizzate al potenziamento dell'individuo.

Per quanto riguarda l'eventuale relazione temporale tra psicoterapia di supporto ed eventuale misura alternativa, la dinamica psicoterapeutica proposta rende auspicabile che l'incontro tra terapeuta e "cliente" si realizzi già durante la detenzione e si proietti nel futuro psicologico, legato anche all'alternativa alla detenzione.

In sostanza, una psicoterapia programmata può avere inizio anche nell'immediatezza di un percorso che si realizzi nell'ambito di una alternativa alla detenzione.

Con questi approfondimenti, si è concluso il progetto relativo alla consulenza tecnica di ufficio che può essere considerata un esempio di perizia criminologica che ha concorso al più ampio progetto che è sfociato nella decisione del Tribunale di Sorveglianza di Bologna.

\section{Ordinanza del Tribunale di Sorveglianza di Bologna.}


Il Tribunale di Sorveglianza di Bologna, nell'ordinanza emessa in data 24 giugno $2014^{13}$, dopo aver considerato in dettaglio tutti gli aspetti di questa vicenda giudiziaria, osserva "che sussistono tutti i presupposti previsti dall'art. 47 quinquies L. n. 354/75, ai fini della concessione dell'invocata misura alternativa 'speciale'.

In primo luogo, non ricorrono, infatti, le condizioni di cui all'art. 47 ter O.P., posto che la pena residua espianda è superiore ad anni quattro di reclusione. Il figlio della condannata, come si è già detto, all'atto della proposizione dell'istanza, aveva età inferiore ai dieci anni.

Per quanto attiene al pericolo di recidiva, la residua pericolosità sociale dell'istante, per come evidenziata anche dalla consulenza tecnica d'ufficio disposta da questo Collegio, pericolosità peraltro generica e non già specifica, affonda le sue radici in un disturbo psicologico di adattamento derivante dagli avvenimenti traumatici che hanno caratterizzato la biografia della detenuta negli ultimi dodici anni: la morte per uccisione del figlio, le vicende giudiziarie, la condanna ritenuta ingiusta, $\mathrm{i}$ dissapori con la famiglia di origine, i conflitti con un difensore e soprattutto, la detenzione.

AMF, per come evidenziato dalle consulenze in atti, non manifesta evidenze di patologie psichiatriche. Presenta, piuttosto, i sintomi di un disturbo dell'adattamento con segni depressivi, tratti di narcisismo ed egocentrismo con idee dominanti, disarmonie che, a parere del consulente tecnico d'ufficio, possono essere adeguatamente trattate con lo strumento della

\footnotetext{
${ }^{13}$ Il Tribunale di Sorveglianza, riunito in Camera di Consiglio, era così composto: Dott. Francesco Maisto Presidente, Dott. Sabrina Bosi - Magistrato di Sorveglianza est, Dott. Cinzia Bertuccioli - esperta, Dott. Stefano Rambelli - esperto.
}

psicoterapia di sostegno, strumento per il quale la condannata dispone di idonee risorse e verso il quale è ben disposta, come dimostrano l'avvenuto contatto con uno psicoterapeuta durante la detenzione e l'esistenza di un programma di sostegno psicoterapeutico.

La condannata ha dato prova di essere consapevole della necessità di un supporto esterno nella gestione della nuova realtà che potrebbe aprirsi con la concessione della speciale misura alternativa. La terapia inciderebbe sulle disarmonie di personalità e sui disturbi dell'umore evidenziati dal C.T.U., aiutando la condannata a superare gli esiti delle vicende traumatiche che ne hanno segnato la biografia negli ultimi dodici anni, consentendole di riappropriarsi pienamente del proprio ruolo di madre e di affrontare le problematiche che dovessero presentarsi nel reinserimento nell'ambiente socio-familiare.

Sembra, peraltro, importante e tranquillizzante per il Tribunale riflettere su due circostanze di fatto, talora omesse in quanto solo apparentemente banali.

In primo luogo, AMF dopo la commissione del reato, ha trascorso un lungo periodo di libertà in ambito familiare senza mettere in atto alcun agito deviante.

In secondo luogo, quelle particolari condizioni, anche ambientali, che fecero da sfondo alla perpetrazione del delitto, non possono riproporsi, per la radicalmente mutata situazione ed ambientazione del nucleo familiare.

Conviene anche sottolineare che la detenzione domiciliare 'speciale', è stata introdotta nell'ordinamento penitenziario dall'art. 3 della Legge n. 40/2001, e che per giurisprudenza costante di legittimità, l'istituto risulta 
'...finalizzato, in presenza di determinati presupposti e circostanze, sia al reinserimento sociale della condannata (alla pari di tutte le misure alternative alla detenzione in carcere), sia a consentire alla detenuta, madre di prole di età non superiore ai dieci anni, di occuparsi della cura e dell'assistenza dei figli minori. Entrambi tali profili devono essere doverosamente considerati dal Giudice che...deve esaminare le possibilità di reinserimento sociale della condannata e di effettivo esercizio delle cure parentali nei confronti dei figli di età non superiore ai dieci anni, quest'ultimo determinando il maggior ambito di applicazione della misura alternativa e l'altro permettendo il regime alternativo alla detenzione...' (cfr. Cass., sez. I, n. 38731/2013).

AMF ha compiuto, nel corso di anni, rilevanti progressi nel trattamento rieducativo, essendo stata progressivamente ed adeguatamente avviata alla sperimentazione di quote sempre maggiori di impegni, di relazioni, di libertà responsabile all'esterno del carcere, mediante la positiva esperienza dei numerosi permessi premiali fruiti, e del quotidiano lavoro all'esterno, dando prova di un progressivo reinserimento nell'ambiente esterno.

La volontà del recupero sociale è, d'altro canto, dimostrata dall'avvenuta presa di contatto da parte della condannata con uno psicoterapeuta con il quale ha concordato un percorso di supporto alla gestione delle eventuali e nuove condizioni personali ed all'esercizio della genitorialità, in particolare nei riguardi del figlio.

Né, d'altro canto, ritiene il Collegio, potrebbe essere considerata ostativa alla concessione della misura alternativa alla detenzione la non ammissione di colpevolezza da parte della condannata.

A tale riguardo, infatti, la giurisprudenza della Corte di Cassazione è univoca nel senso che, ai fini dell'ammissione alle misure alternative, non è richiesta la confessione del condannato, ‘...il quale ha il diritto di non ammettere le proprie responsabilità, pur dovendosi attivare per prendere parte in modo attivo all'opera di rieducazione... (cfr. Cass., sez. I, n. 13445/2013).

Ancora: '...la mancata ammissione da parte del condannato della propria colpevolezza non può indurre ad una prognosi sfavorevole in ordine alla commissione di altri reati, sia perché nel processo penale l'imputato non ha obbligo di verità, sia perché l'assenza di confessione può essere dettata dai più svariati motivi senza che, solo per questo, essa sia sintomatica di mancato ravvedimento o di pericolosità sociale o dell'intenzione di persistere nel crimine...' (cfr. Cass., sez. I, n. 33287/2013).

L'atteggiamento di negazione dell'addebito, ritiene la Corte, deve essere sempre preso in considerazione, in un contesto di analisi globale della personalità individuale e di verifica della sua evoluzione psicologica, al fine di verificare se il condannato abbia prestato collaborazione al processo rieducativo.

Si può pertanto sostenere che sia avvenuta una evoluzione della personalità della condannata, nel senso di una manifestata adesione ai valori della civile convivenza e di una attiva adesione al percorso rieducativo.

I progressi avvenuti nel corso del trattamento, anche mediante la positiva e prolungata sperimentazione in ambiente esterno, depongono 
per la sussistenza di condizioni favorevoli al reinserimento all'esterno, attraverso opportuni interventi di sostegno, già attivati, mediante l'invocata misura alternativa.

Peraltro, l'ambiente socio-familiare, per come descritto nelle numerose relazioni dei servizi sociali in atti, appare ampiamente favorevole ad accogliere la condannata ed a sostenerla.

La coppia viene descritta come coesa, legata da affetto sincero e reciproca fiducia e capace di ben gestire gli anni, difficili, della detenzione della donna, creando una vasta rete di supporto e garantendo ai figli un ambiente sereno, adeguato alle loro esigenze e protettivo.

Passando quindi all'esame dell'ulteriore parametro cui risulta finalizzato il beneficio penitenziario invocato, ovvero la possibilità di ripristinare la convivenza con il figlio, occorre evidenziare come le indagini affidate al servizio sociale del Comune di residenza della famiglia, dal locale Tribunale per i minorenni abbiano accertato '...che $\mathrm{i}$ due minori vivono in un ambiente protetto e tutelato dai genitori e che gli stessi sono curati ed accuditi adeguatamente sia sotto il profilo pratico che sotto il profilo emotivo...' (v. decreto del 6/04/2006).

Dalle relazioni del servizio sociale, in atti, emerge come la coppia genitoriale sia sempre stata coesa ed attenta ai bisogni del minore. AMF, compatibilmente con la propria condizione detentiva, ha sempre dedicato tutto il tempo consentitole alla cura dei figli, mostrandosi in grado di coglierne i bisogni e le problematiche, ed attivandosi come madre al fine di promuoverne il benessere, mantenendo una forte presenza emotiva e psicologica, nonché educativa verso i figli, in stretta collaborazione con il marito. In particolare, il figlio minore risulta soffrire, dal punto di vista psicologico e dell'andamento scolastico, per l'assenza della madre, manifestando sintomi di disagio e difficoltà nell'apprendimento scolastico.

$\mathrm{Si}$ può ritenere che la concessione dell'invocata misura alternativa, ripristinando la convivenza tra il bambino e la condannata, si risolverebbe certamente in favore del minore, il quale manifesta segni rilevanti di sofferenza e disagio dovuti all' assenza fisica della madre, con rilevante compromissione dell'andamento scolastico e disagi psicologici evidenti.

All'esito del complesso e globale scrutinio favorevole alla concessione della detenzione domiciliare alla condannata, il Tribunale, come previsto dal comma 3 dell'art. 47 quinquies O.P., impone le opportune prescrizioni relative ai necessari interventi del servizio sociale, finalizzate a favorire il reinserimento della condannata, un adeguato esercizio della funzione genitoriale, nonché a tutela del minore.

P.Q.M.

Visti gli artt. 47 ter, quinquies e 70 O.P., 284 c.p.p.

ammette AMF alla misura della detenzione domiciliare per tutto il periodo residuo di pena da espiare.

Ne ordina, pertanto, l'immediata scarcerazione dalla Casa Circondariale di Bologna, se non detenuta per altra causa, per raggiungere, libera nella persona, il domicilio sotto individuato.

LA DETENZIONE DOMICILIARE HA INIZIO DAL GIORNO DELLA NOTIFICA DELLLA PRESENTE ORDINANZA APPENA DEPOSITATA IN CANCELLERIA 
Il detenuto domiciliare dovrà osservare le seguenti:

\section{prescrizioni}

a) obbligo di non allontanarsi dal luogo della detenzione domiciliare [...];

salve le eccezioni che seguono:

- visto l'art. 47 ter, o.p. il detenuto domiciliare potrà recarsi presso ambulatori, servizi sanitari e ospedalieri, per interventi, accertamenti diagnostici e cure, previa comunicazione di luoghi e tempi di spostamento all'U.E.P.E. e all'Autorità di Vigilanza competenti. In caso di ricovero per motivi sanitari la detenzione domiciliare dovrà intendersi eseguita nel luogo di attuazione dello stesso, che dovrà essere sollecitamente reso noto all'U.E.P.E. e all'Autorità di Vigilanza, i quali a loro volta ne daranno tempestiva informazione al Magistrato di Sorveglianza competente;

- la condannata potrà allontanarsi dal domicilio ogni giorno per ore quattro, $d a$ concordare con l'Autorità di Vigilanza, nell'ambito della Provincia di Bologna, al fine di soddisfare esigenze di vita legate esclusivamente alla gestione del nucleo familiare;

b) divieto di detenere armi e stupefacenti;

c) divieto di assumere sostanze stupefacenti ed abusare di bevande alcoliche;

d) obbligo di recarsi una volta la settimana a Bologna per esigenze legate all'attività lavorativa, concordando giorno ed orari con l'Autorità di Vigilanza;

e) divieto di frequentare pregiudicati e/o tossicodipendenti; f) divieto di frequentare persone estranee ai familiari elo conviventi;

g) divieto di allontanarsi dal territorio della Provincia di Bologna e quindi di recarsi in altre zone del territorio nazionale con particolare riferimento a Cogne.

Dispone trasmettersi copia della presente ordinanza all'UEPE di Bologna, incaricato, nei confronti della condannata, dei seguenti interventi:

a) effettuerà, tramite assistente sociale incaricata, visita settimanale presso l'abitazione della condannata;

b) verificherà l'assiduità delle sedute di psicoterapia effettuate dalla condannata;

c) verificherà se l'assistente sociale incaricato della vigilanza sulle condizioni del minore si rechi periodicamente presso il nucleo familiare della condannata.

L'UEPE di Bologna relazionerà mensilmente al Magistrato di Sorveglianza sull'andamento della misura alternativa.

Demanda la vigilanza sull'esecuzione della misura alla Stazione Carabinieri [...].

Dispone trasmettersi gli atti al Magistrato di Sorveglianza di Bologna.

Bologna, 24-06-2014".

Contro l'ordinanza del Tribunale di Sorveglianza del 24 giugno 2014, la Procura generale della Repubblica presso la Corte d'Appello di Bologna il 3 luglio 2014 ha proposto ricorso alla Suprema Corte di Cassazione perché la suddetta ordinanza venisse annullata.

La Procura generale presso la Corte di Cassazione in data 10 ottobre 2014 ha chiesto che la Corte di Cassazione rigettasse il suddetto ricorso. 
La Corte di Cassazione in data 11 febbraio 2015 ha annullato l'ordinanza impugnata e ha rinviato per nuovo esame al Tribunale di Sorveglianza di Bologna.

Il ricorso della Procura generale presso la Corte di Appello di Bologna, che appunto partecipa alle udienze del Tribunale di Sorveglianza con proposizioni di pareri e con poteri di ricorrere in Cassazione, denota come appaia complessa la materia trattata anche nel caso studiato, così che assume importanza, come possibile oggetto di ulteriore approfondimento e di studio, la decisione della Corte di Cassazione che ha annullato con rinvio l'ordinanza del Tribunale di Sorveglianza di Bologna e che ha pronunciato la sentenza ${ }^{14}$ di cui, qui di seguito, si riportano alcuni brani:

"1. Il Tribunale di sorveglianza di Bologna, con ordinanza del 24 giugno 2014, ammetteva AMF alla misura della detenzione domiciliare speciale di cui all'art. 47-quinquies 1. 354/1975 per il residuo pena da espiare, pari, a quella data, ad anni tredici di reclusione.

A sostegno della decisione il tribunale, verificata preliminarmente l'ammissibilità della domanda e richiamata la condanna in espiazione a sedici anni di reclusione per aver ucciso il proprio figlio di anni tre, [...], richiamava: la osservazione scientifica della personalità della condannata redatta presso la Casa circondariale [...], la relazione dell'esperto psicologo di cui all'art. 80 O.P., l'ammissione, a far tempo dal mese di ottobre 2013, al lavoro esterno, la perizia medicolegale disposta dal tribunale il 21.1.2014 al fine di valutare il grado di pericolosità sociale della

${ }^{14}$ Corte Suprema di Cassazione - I Sezione Penale (Presidente Dott. Arturo Cortese - Consigliere Relatore Dott. Francesco Maria Silvio Bonito) - Sentenza ${ }^{\circ}$ $354 / 2015$ del 11/2/2015. detenuta, perizia arricchita dai test di Rorschach, MMPI-2 e Bender, la consulenza tecnica di ufficio, che ha registrato una PCL (psychopathy checklist-revised) favorevole alla periziata, il supplemento di indagine di ufficio disposto dal tribunale all'udienza del 29 aprile 2014, la relazione del Servizio sociale [...] sui figli della detenuta ed, in generale, sulla situazione del suo nucleo familiare e, sulla base dei relativi esiti, puntualmente sintetizzati e criticamente valutati, ha concluso per l'accoglimento della istanza della detenuta sul duplice presupposto dell'assenza di una situazione di pericolosità sociale specifica, di una situazione ampiamente comprovata di difficoltà esistenziale del figlio [minore] riferibile all'assenza fisica della madre e dell'utilità della misura al ripristino della convivenza con la mamma.

2. Ricorre per cassazione avverso detto provvedimento il Procuratore generale della repubblica di Bologna contestandone la legittimità per vizio della motivazione e violazione di legge, in particolare osservando: non ricorre nella fattispecie uno dei requisiti richiesti dalla legge per la legittima adozione della misura in discussione, giacchè, allo stato, il figlio [minore], alla cui cura la misura sarebbe finalizzata, ha un'età superiore ad anni dieci [...].

3. Con argomentata requisitoria scritta il P.G. in sede ha concluso per il rigetto del ricorso, rilevando l'ammissibilità della domanda difensiva e la esaustiva logicità della motivazione articolata dal tribunale.

[...]

4.3. [...] Dalle esposte considerazioni consegue che l'ordinanza impugnata si appalesa illegittima nella parte in cui, dopo aver correttamente 
delibato la sussistenza delle condizioni richieste dalla legge in relazione al momento della presentazione iniziale della domanda, non ha tenuto conto che, essendo stato superato, al momento della decisione, il limite dei dieci anni del minore, era necessario, per dare positivo corso alla misura, di fatto divenuta soggetta a prorogabilità contestualmente alla sua (tardiva) concessione, verificare la sussistenza dei (diversi) requisiti cui la legge subordina la concedibilità della proroga.

5. L'ordinanza impugnata va pertanto annullata con rinvio al giudice a quo affinché riesamini la domanda proposta dalla detenuta alla luce dell'ulteriore, seguente principio di diritto: 'in tema di detenzione domiciliare speciale di cui all'art 47-quinquies O.P., qualora tra la domanda della detenuta ed il momento della decisione muti il requisito dell'età del figlio (che in tale periodo raggiunge il decimo anno di età) il tribunale di sorveglianza adito dovrà considerare la domanda principale alla stregua, altresì, di una domanda di proroga della misura eppertanto valutare la ricorrenza non soltanto dei requisiti richiesti per il riconoscimento della misura dal primo comma dell'art. 47-quinquies, bensì anche quelli di cui al successivo ottavo comma, giacché è da considerarsi illegittima la misura in discorso in costanza di prole superiore ad anni dieci se non ricorrenti i requisiti di cui a detto comma'.

\section{P.T.M.}

la Corte annulla l'ordinanza impugnata e rinvia per nuovo esame al Tribunale di sorveglianza di Bologna.

Così deciso in Roma, addì 11 febbraio 2015”.

E' evidente che, dalla dialettica tra organi ed uffici giudiziari, emerge la complessità della gestione della pena tra detenzione e misure alternative alla detenzione stessa. Perciò appaiono attuali tutti gli insegnamenti del Beccaria sulla certezza e sulla severità della pena ${ }^{15}$ e risulta ancora significativo il richiamo di Marc Ancel, che su queste questioni, nella seconda metà degli anni Settanta del secolo scorso, precisava: "siamo in un'epoca nella quale molti e molti dubbi sono stati espressi sull'efficacia se non anche sulla legittimità della detenzione. È un'epoca nella quale i metodi di trattamento in libertà sono stati incessantemente sviluppati; e nella quale è essenziale che il giudice abbia un campo flessibile di sanzioni a sua disposizione" ${ }^{" 16}$.

Le problematiche connesse infatti all'ordinamento penitenziario sono l'esempio emblematico di quelle questioni che vanno poste in termini molto ampi, fino ad interessare la responsabilità degli enti locali e statuali competenti. A questo proposto, occorre ricordare l'importanza delle istituzioni a cui è affidata la ricerca e l'insegnamento universitario: in effetti, è tempo che la criminologia prenda sempre più coscienza del fatto che criminalità, devianza e pena sono un complesso problema sociale e politico. Nell'ambito quindi di queste problematiche, non si può trascurare il ruolo della ricerca: a questo proposito, può essere ancora significativa l'analogia usata da Donald Cressey, già nel 1964, che, durante la "Arden House Conference on Correctional Manpower on Training", tracciò un parallelo tra divisione del lavoro nel programma spaziale e nel campo dei sistemi "di correzione"17,

15 Beccaria C., Dei delitti e delle pene. Consulte criminali, i grandi libri Garzanti, Milano, 1989.

16 Ancel M., La sospensione dell'esecuzione della sentenza, Etas Libri, Milano, 1976, p. 85.

${ }^{17}$ Waldo C.P., "Research on Training in Corrections: the Role of the University", Federal Probation, June 
mettendo in evidenza l'importanza di questo settore per quanto riguardava anche il suo inserimento nel bilancio di uno stato.

Pertanto, alla ricerca deve essere consentito di avere un ruolo importante sia nei riguardi della criminalità e del trattamento degli autori di reato, sia nella formazione degli operatori sociali che svolgono la loro attività nel settore della prevenzione e del trattamento: in questa prospettiva si colloca questo studio.

\section{Ulteriori riflessioni.}

Nell'ambito di questa indagine, si è affrontato il problema di accertare quale sarebbe stato il comportamento futuro di una persona, cioè se fosse probabile che questi potesse commettere nuovi fatti preveduti dalla legge come reati. Si tratta di una questione antica che riguarda le ricerche scientifiche sulla personalità: se queste ricerche avessero un esito positivo, si avrebbe come risultato finale la capacità di predire con esattezza le irregolarità della condotta umana. $\mathrm{Ne}$ consegue che tale capacità consentirebbe di esercitare un controllo sul comportamento stesso: infatti si concede una misura alternativa alla detenzione previo accertamento che colui che ha commesso il fatto non sia persona socialmente pericolosa. Ciò comporta che la capacità di prevedere il futuro comportamento di un individuo si collega alla capacità di controllare il comportamento medesimo e quindi tutto ciò farà sì che si ripresenti l'interrogativo riguardante $c h i$

1971, p.57; Emeri A., Balloni A., "Struttura e finalità dei trattamenti in libertà degli autori di reato", Studi di Sociologia, Anno XI (1973), fascicolo I-II, pp. 147159; Balloni A., "Nuovo ordinamento penitenziario. Osservazioni storico-politico-sociali", Relazione tenuta al "Convegno nazionale sul nuovo ordinamento penitenziario", Camerino, 11-12 dicembre 1976, Clueb, Bologna, 1977. controllerà il comportamento degli esperti, problema per lo meno vecchio quanto Giovenale: quis custodiet custodes ipsos?

Queste considerazioni devono collegarsi a quel tentativo di superamento di un procedimento astrattamente classificatorio e devono tendere ad un metodo costruttivo, essenzialmente concreto. Anche in questo caso, l'interesse non doveva essere prevalente per gli aspetti percettivi, comune a molti eventi vissuti come fra loro simili, ma si doveva portare l'attenzione sul rapporto che intercorre tra il verificarsi di diversi eventi e la presenza, nell'ambiente in cui si sviluppano, di determinate concrete condizioni. In una tale prospettiva, si è fatto riferimento al colloquio che è quella particolare situazione "in cui la comunicazione avviene in primo luogo a voce, in un gruppo di due persone, che si incontrano più o meno volontariamente" ${ }^{\text {18. }}$.

È evidente che nel caso in esame si è verificata una variazione rispetto a questa definizione: infatti il colloquio non avveniva per una scelta volontaria, ma era legato al fatto che si sviluppava in una dinamica legata appunto alla consulenza tecnica in cui gli incontri erano caratterizzati dalla presenza della condannata, dal suo consulente di parte e dal consulente d'ufficio, quindi non c'era volontarietà e vi erano due persone osservanti e un osservato. La persona osservata era stata invitata all'osservazione a seguito della disposizione della consulenza tecnica d'ufficio e i due osservatori avevano ruoli diversi: uno era il consulente dell'ufficio, l'altro il consulente dell'osservata. Tuttavia si è realizzato una buona atmosfera di incontro, che ha dato la possibilità di far sì che il colloquio non fosse influenzato da quei fattori che 
possono distorcerne l'andamento, quali la sfiducia o la diffidenza e soprattutto l'influenza della personalità dell'esaminatore che può suscitare emozioni e reazioni particolari nell'osservato. La presenza del consulente tecnico di parte, che già conosceva la condannata, può essere considerato sul piano clinico un elemento eccentrico o abnorme, ma nell'ambito psicopatologico forense questa presenza, se ricondotta nell'ambito di un'obiettiva e leale collaborazione, può far superare quell'aspetto importante che è il livello di diffidenza per cui si realizza una situazione completamente diversa rispetto a quanto si verifica allorché il colloquio avviene a fini clinico-diagnostici. In tal caso può verificarsi una situazione di complicità per cui il clinico è percepito come una persona dotata di grandi capacità che può risolvere un problema diagnostico e terapeutico. Nell'ambito psicopatologico forense o criminologicopsichiatrico, si tratta di realizzare una conoscenza per trasferirla sulle decisioni: in tal caso consulente d'ufficio e consulente di parte collaborano in questa prospettiva e questa collaborazione nel caso in esame si è realizzata, producendo effetti positivi nell'ambito di quel progetto operativo che era la relazione di consulenza tecnica d'ufficio, che va immessa in un più ampio progetto operativo che riguarda appunto il progetto rieducativo.

Da questi presupposti, facendo ricorso alla psicologia topologica di Kurt Lewin, è evidente che il comportamento della persona è in rapporto all'ambiente e di questo fanno parte gli esaminatori: il comportamento della condannata si legava al comportamento degli esaminatori e

\footnotetext{
${ }^{18}$ Sullivan H.S., Il colloquio psichiatrico, Feltrinelli,
}

questi ultimi traevano gli elementi necessari dai colloqui per la previsione del comportamento futuro e del rischio di recidivismo ${ }^{19}$.

I numerosi colloqui hanno rappresentato il fulcro dell'attività di consulenza, ma ad essi vanno collegati i risultati delle analisi del contenuto della documentazione clinica, giudiziaria e penitenziaria e l'apporto dei reattivi mentali realizzati dal collaboratore del consulente d'ufficio e dal collaboratore del consulente di parte. È evidente che nell'ambito psicopatologico forense $o$ in quell'ambito che può essere definito di criminologia psichiatrica, ogni caso assume una valenza particolare per cui potrebbe essere pericoloso legarsi strettamente a classificazioni o a schemi statistici perché, allorché si fanno previsioni riguardanti il comportamento futuro, si dovrebbe conoscere la personalità dell'autore di un crimine, intesa come comportamento globale che si apprende appunto anche da una buona capacità di realizzare la storia di vita collegandola alle documentazioni che vengono fornite da varie fonti.

In questa esperienza che si potrebbe definire progetto realizzato di perizia criminologica, un dato emerge: vi è la necessità, tenendo conto dell'individualizzazione del trattamento, di sviluppare gli accertamenti nell'ambito criminologico-psichiatrico centrandoli sulla storia di vita del condannato e sulle sue caratteristiche di personalità, soprattutto quando si opera su quella linea rieducativa che tende a valorizzare il

\footnotetext{
Milano, 1975, p. 4.

${ }^{19}$ Balloni A., Sabattani P.G., "Considerazioni sul ruolo del criminologo nelle strutture penitenziarie", Critica Penale, F. 1, 1971; Balloni A., "Criminogenesi e rieducazione”, Giovani Realtà, A. VII, n. 24, 1987, pp. 25-57.
} 
trattamento, dando un'attenzione costante

all'individuo nel suo ambiente di vita.

\section{Bibliografia.}

- American Psychiatric Association, DSM-IV$T R$, Manuale diagnostico e statistico dei disturbi mentali, Masson, Milano, 2003.

- Ancel M., La sospensione dell'esecuzione della sentenza, Etas Libri, Milano, 1976.

- Balloni A., Sabattani P.G., "Considerazioni sul ruolo del criminologo nelle strutture penitenziarie", Critica Penale, F. 1, 1971.

- Balloni A., "Nuovo ordinamento penitenziario. Osservazioni storico-politicosociali", Relazione tenuta al "Convegno nazionale sul nuovo ordinamento penitenziario", Camerino, 11-12 dicembre 1976, Clueb, Bologna, 1977.

- Balloni A., "Colloquio, test mentali e psicopatologia", in Guidicini P. (a cura di), Nuovo manuale della ricerca sociologica, Franco Angeli, Milano, 1981, cap. 14, pp. 339-351.

- Balloni A., Criminologia in prospettiva, Clueb, Bologna, 1983.

- Balloni A., "Criminogenesi e rieducazione", Giovani Realtà, A. VII, n. 24, 1987, pp. 2557.

- Balloni A., "La perizia psichiatrica: problemi, ipotesi e prospettive", in Balloni A., (a cura di), Vittime Crimine Difesa Sociale, Clueb, Bologna, 1989, pp. 51-73.

- Balloni A., "Nuove metodologie nell'indagine peritale con riferimento alla psicopatologia", in Lombardi M.A. (a cura di), La Perizia e la Consulenza tecnica: stato attuale $e$ prospettive, Pacini Editore, Pisa, 1990.

- Balloni A., "Psichiatria forense", in Pancheri P. e Cassano G.B. (coordinato da), Trattato italiano di psichiatria, Masson, Milano, 1999.

- Balloni A., Criminologia e psicopatologia. Analisi di 110 perizie psichiatriche, Patron, Bologna, 2004.
- Balloni A., "La teoria del campo di Kurt Lewin e le sue applicazioni in criminologia", Rivista di Criminologia, Vittimologia $e$ Sicurezza, Vol. III, N.3 - Vol. IV - N.1, Settembre 2009 - Aprile 2010, pp. 171-186.

- Balloni A., Nathan F., "Il colloquio clinico nella perizia psichiatrica", Giornale $D i$ Medicina Legale Infortunistica E Tossicologia, Suppl. al n. 4,1968, pp. 636644.

- Balloni A., Bisi R., Sette R., Manuale di Criminologia - Volume I - Le Teorie, Clueb, Bologna, 2013.

- Balloni A., Bisi R., Sette R., Manuale di Criminologia - Volume II - Criminalità, Controllo, Sicurezza, Clueb, Bologna, 2013.

- Beccaria C., Dei delitti e delle pene. Consulte criminali, i grandi libri Garzanti, Milano, 1989.

- Emeri A., Balloni A., "Struttura e finalità dei trattamenti in libertà degli autori di reato", Studi di Sociologia, Anno XI (1973), fascicolo I-II, pp. 147-159.

- Galli G. (a cura di), La persona in relazione. Sviluppi della psicologia della Gestalt, Liguori Editore, Napoli, 2009.

- Pancheri P., Brugnoli R., Marconi P.L., Carilli L, "Pensiero", in Pancheri P., Cassano G.B. (coordinato da), Trattato italiano di psichiatria, vol. I, Masson, Milano, 1999, pp.547-548.

- Rogers C.R., La terapia-centrata-sul -cliente, G. Martinelli Editore, Firenze, 1970.

- Strzyz K., Narcisismo e socializzazione. Trasformazione sociale e il mutamento di dati caratteriali, Feltrinelli, Milano, 1981.

- Sullivan H.S., Il colloquio psichiatrico, Feltrinelli, Milano, 1975.

- Waldo C.P., "Research on Training in Corrections: the Role of the University", Federal Probation, June 1971. 